Research Paper

\title{
The Assessment of Tumor Response by Measuring the Single Largest Lesion per Organ in Metastatic Tumors: A Pooled Analysis of Previously Reported Data
}

\author{
Hyun Joo Jang ${ }^{*}$, Ji Woong Cho²*, Bumjung Park ${ }^{3}$, Hyun Chang Choi ${ }^{4}$, Hyeong Su Kim4, Jung Han Kim ${ }^{\bowtie}$ \\ 1. Department of Internal Medicine, Dongtan Sacred Heart Hospital, Hallym University Medical Center, Hallym University College of Medicine, \\ Hwasung 445-907, Republic of Korea \\ 2. Department of Surgery, Hallym University Sacred Heart Hospital, Hallym University Medical Center, Hallym University College of Medicine, \\ Anyang 431-070, Republic of Korea \\ 3. Department of Otolaryngology-Head and Neck Surgery, Hallym University Sacred Heart Hospital, Hallym University Medical Center, Hallym \\ University College of Medicine, Anyang 431-070, Republic of Korea \\ 4. Department of Internal Medicine, Kangnam Sacred Heart Hospital, Hallym University Medical Center, Hallym University College of Medicine, \\ Seoul 150-950, Republic of Korea \\ * Hyun Joo Jang and Ji Woong Cho equally contributed to this work.
}

\begin{abstract}
$\triangle$ Corresponding author: Jung Han Kim, MD, PhD, Division of Hematology-Oncology, Department of Internal Medicine, Kangnam Sacred Heart Hospital, Hallym University Medical Center, Shingil-Ro 1, Youngdeungpo-Gu, Seoul 150-950, South Korea. E-mail: harricil@hotmail.com, harricil@hallym.or.kr

(c) Ivyspring International Publisher. This is an open-access article distributed under the terms of the Creative Commons License (http://creativecommons.org/ licenses/by-nc-nd/3.0/). Reproduction is permitted for personal, noncommercial use, provided that the article is in whole, unmodified, and properly cited.
\end{abstract}

Received: 2014.10.26; Accepted: 2014.12.08; Published: 2015.01.05

\begin{abstract}
Background: The RECIST 1.1 adopted a total of five target lesions to be measured, with a maximum of two lesions per organ. To the best of our knowledge, the criterion of two target lesions per organ in the RECIST 1.1 is arbitrary and has not been supported by any objective evidence. Recently, we reported that the modified RECIST 1.1 (measuring the single largest lesion in each organ) showed a high level of concordance with the original RECIST 1.1 in patients with advanced or metastatic non-small cell lung cancer (NSCLC), gastric cancer (GC), and colorectal cancer (CRC). However, each study had a major limitation of a small number of patients.

Methods: We conducted a pooled analysis using the data from the three individual studies to improve statistical power. Tumor responses were compared according to the RECIST 1.1 and modified RECIST 1.1 (mRECIST 1.1).

Results: A total of 153 patients who had at least two target lesions in any organ according to the RECIST 1.1 were included in this pooled study: 64 with NSCLC, 51 with GC, and 38 with CRC. Regardless of primary sites, the number of target lesions according to the mRECIST 1.1 was significantly lower than that according to the RECIST $1.1 \quad(P<0.001)$. The assessment of tumor responses showed a high concordance between the two criteria $(k=0.908)$. Only eight patients (5.2\%) showed disagreement in the tumor response assessment between the two criteria. The overall response rates of chemotherapy were not significantly different between the two criteria (33.3\% versus $33.3 \%, P=1.0$ ).

Conclusions: The modified RECIST 1.1 was comparable to the original RECIST 1.1 in the tumor response assessment of patients with advanced or metastatic NSCLC, GC, and CRC. Our results suggest that it may be possible to measure the single largest lesion per organ for assessing tumor response in clinical practice.
\end{abstract}

Key words: RECIST 1.1; modified RECIST 1.1; Target lesion; Tumor response; Single-lesion measurement 


\section{Introduction}

Since the early 1980s, the World Health Organization (WHO) has adopted $\mathrm{WHO}$ response criteria as a standard method for evaluating tumor response [1]. According to the WHO criteria, total tumor burden is determined bi-dimensionally by the sum of the products of the two longest diameters in the perpendicular dimensions of all target lesions. The criteria for both response and progression are expressed as percentage changes in the sum of tumor measurements from baseline. Since the details for selecting and measuring target lesions were not clearly described in the WHO guidelines, however, the assessment of tumor responses has shown a high intra- and inter-observer variability [2, 3]. In addition, measuring all target lesions in two dimensions and then calculating the sums of their products is not only time-consuming in clinical practice but also has a potential risk of measurement errors.

In 2000, the Response Evaluation Criteria in Solid Tumors (RECIST) Working Group proposed the RECIST guideline version 1.0 (RECIST 1.0) to simplify and clarify tumor response criteria [4]. Major features of the RECIST 1.0 included the definition of minimum size of measurable lesions by computed tomography (CT), the use of uni-dimensional measures instead of bi-dimensional method for the overall evaluation of tumor burden, and the instruction on how many target lesions to be measured. The RECIST 1.0 criteria adopted a total of 10 target lesions with a maximum of 5 lesions per organ. It has been widely accepted as a standardized method for tumor response assessment, especially in clinical trials with objective response or time to progression as primary end points. A number of issues and questions concerning the number of target lesions, the size of lymph nodes (LNs) to be measured, and the application of new imaging technologies such as multi-detector CT (MDCT) and positron emission tomography (PET), however, has been newly raised on the RECIST $1.0[5,6]$.

In 2009, based on the analyses of the vast database of more than 6,500 patients with over 18,000 target lesions from 16 clinical trials, the RECIST Working Group presented a revised RECIST guideline version 1.1 (RECIST 1.1) to overcome the limitations of the original RECIST $1.0[6,7]$. The significant changes in the RECIST 1.1 included the update concerning maximum number of target lesions, the LN measurement, the clarification of disease progression, and the inclusion of PET/CT in the detection of new lesions [6-9]. Especially, the maximum number of target lesions to be assessed has been reduced from ten to five in total, and from five to two target lesions per organ with metastases. While the total of ten tar- get lesions in the RECIST 1.0 was arbitrarily selected, the RECIST 1.1 defined a total of five lesions through the patients' data analysis [8] and statistical simulating studies $[10,11]$. To the best of our knowledge, however, the criterion of two target lesions per organ was an arbitrary decision and has not been supported by any objective evidence. Therefore, the optimal number of target lesions per organ to accurately evaluate tumor response still needs to be determined in further studies. Under the conditions of accurately measuring the changes of tumor burden, it is desirable to simplify the international guidelines for assessing tumor response as far as possible.

Before the RECIST 1.1 was presented, Zacharia et al. had reported that measuring the single largest hepatic lesion yielded the same response classification as measuring up to five target lesions in the majority of patients with liver metastases of colorectal cancer (CRC) [12]. Based on this finding, we hypothesized that measuring the single largest lesion in each organ (modified RECIST 1.1; mRECIST 1.1) might show almost the same response classification as measuring two target lesions per organ (RECIST 1.1) and compared successively the tumor responses by CT between the RECIST 1.1 and mRECIST 1.1 in patients with advanced or metastatic non-small cell lung cancer (NSCLC) [13], gastric cancer (GC) [14], and colorectal cancer (CRC) [15]. We found that, with a decreased total number of target lesions to be measured, the mRECIST 1.1 was comparable to the original RECIST 1.1 in the assessment of tumor responses. However, each study had a major limitation of a small number of patients. In this study, we conducted a pooled analysis using data from the previously reported three studies [13-15] to improve statistical power.

\section{Materials and Methods}

\section{Patients}

Each study obtained the Institutional Review Board's approval with a waiver of patients' informed consent according to the Korean Ethical Guidelines for epidemiological researches. We reviewed the medical records of patients with advanced or metastatic NSCLC, GC, and CRC who received a first-line chemotherapy between January 2004 and December 2013 at the Hallym University Medical Centers (Seoul, South Korea). Patients were eligible for the study if they had the following criteria; histologically confirmed adenocarcinoma of the stomach or colon/rectum, or non-small cell carcinoma of the lung, radiologically or histologically confirmed metastatic (in GC and CRC) or advanced (stage IIIB or IV, in NSCLC) disease, having at least two measurable le- 
sions in any organ by the RECIST version 1.1, no history of other cancer, no history of previous chemotherapy or radiotherapy except for adjuvant treatment, and CT tumor assessments at baseline and after chemotherapy. Patients who had shown the development of new lesions or substantial progression of non-target lesions at the follow-up CT were excluded from the final analyses because they would show the same response classification in both criteria. For patients with metastatic CRC, we excluded three patients who had developed new lesions from the original study [15] and newly included another three eligible patients who had received a first-line chemotherapy between July and December, 2013.

\section{CT examinations}

The CT scans for evaluating tumor response were obtained at base line and after about 2 months after the initiation of first-line chemotherapy. All CT examinations were performed on a 64-MDCT scanner (SOMATOM Sensation 64; Giemens Healthcare, Forchheim, Germany) with the administration of an intravenous contrast medium, iopromide (Ultravist 300, Bayer Medical Systems, Berlin, Germany). Especially, the scanning delay was 25 seconds after the initiation of a contrast injection for hepatic artery phase imaging and 65 seconds for portal venous phase imaging. The images were reconstructed with a slice thickness of $5 \mathrm{~mm}$ and were directly uploaded onto the Picture Archiving and Communication System (PACS) workstation (PiView Star, INFINITT Healthcare Co. LTD., Seoul, Korea).

\section{Tumor measurements}

We re-evaluated each patient's tumor measurements from the original CT images. CT tumor measurements were performed manually on axial CT image planes using calipers of a measurement tool on the PACS. The target lesion description and size measurement, the sum of the longest diameters of target lesions, the description of non-target lesions, the development of new lesions, and the tumor response for each patient were recorded by the consensus of two experienced investigators according to the RECIST 1.1 and mRECIST 1.1, respectively. For cases revealing a significant discrepancy between the two investigators, a board-certified chest or abdomen radiologist re-evaluated the $\mathrm{CT}$ measurements.

Briefly, the longest diameters of target lesions were always measured in the axial plane. If lesions on the follow-up CT broke into separate fragments, the sum of the fragments was added, and if lesions coalesced into a single one, the longest diameter of the merged lesion was measured. According to the RECIST 1.1, lytic or mixed lytic-blastic bone lesion with an identifiable soft tissue component was regarded as a target lesion if the soft tissue component meets the criteria of measurability. LN measurements were performed along its short axis according to the RECIST 1.1 criteria, defining LNs of at least $15 \mathrm{~mm}$ as target lesions. LNs with at least $10 \mathrm{~mm}$ but less than $15 \mathrm{~mm}$ along its short axis were regarded as non-target lesions, and LNs with a short axis of less than $10 \mathrm{~mm}$ were regarded as normal. The maximum number of target lesions to be assessed was five in total, with a maximum of two per organ (RECIST 1.1) or a single largest lesion in each organ (mRECIST 1.1).

\section{Definitions of tumor responses}

Patients received a variety of regimens as a first-line chemotherapy in practical setting, and the CT scans for evaluating tumor response were obtained at baseline and after 2 - 4 cycles of the first-line chemotherapy. Because the primary aim of this study was to evaluate the level of concordance of the two criteria, tumor responses were determined with no interval confirmation. The definitions of treatment response were in accordance with the original RECIST version 1.1. Complete response (CR) was defined as the complete disappearance of all tumor lesions. Partial response (PR) was defined as a reduction in the sum of tumor measurements by at least $30 \%$. Progressive disease (PD) was defined as at least $20 \%$ increase in the sum of tumor measurements. The development of new lesions or substantial progression of non-target lesions also was defined as PD. In addition, an absolute increase of at least $5 \mathrm{~mm}$ was a prerequisite for $\mathrm{PD}$. All other forms of tumor response were classified as stable disease (SD).

\section{Statistical analysis}

A paired Student's $t$ test was used to estimate the statistical significance of changes in the number of target lesions at baseline between the RECIST 1.1 and mRECIST 1.1. The Chi-square test was used to compare the overall response rate (ORR) between two groups. A $P$ value of less than 0.05 was considered to indicate a statistical significance. The level of concordance of the tumor responses between the two criteria was estimated using карра statistics. A kappa value of more than 0.75 was regarded as showing strong agreement.

\section{Results}

\section{Patient characteristics}

Patients' baseline characteristics are summarized in Table 1 according to the primary site. There were a total of 153 patients who met the inclusion criteria: 64 with NSCLC [13], 51 with GC [14], and 38 with CRC [15]. 
Table 1. Characteristics of the patients according to the primary sites [13-15].

\begin{tabular}{|c|c|c|c|}
\hline \multirow[t]{2}{*}{ Characteristics } & $\operatorname{NSCLC~}(n=64)$ & $\mathrm{SC}(\mathrm{n}=51)$ & CRC $(n=38)$ \\
\hline & no. $(\%)$ & no. $(\%)$ & no. $(\%)$ \\
\hline Mean age, years (range) & $62.0(29-89)$ & $62.0(31-85)$ & $60.5(42-78)$ \\
\hline \multicolumn{4}{|l|}{ Gender } \\
\hline Male & $49(76.6)$ & $41(80.4)$ & $23(60.5)$ \\
\hline Female & $15(23.4)$ & $10(19.6)$ & $15(39.5)$ \\
\hline \multicolumn{4}{|l|}{ Stage } \\
\hline IIIB & $5(7.8)$ & 0 & 0 \\
\hline IV & $59(92.2)$ & $51(100)$ & $38(100)$ \\
\hline \multicolumn{4}{|l|}{ Histology } \\
\hline Adenocarcinoma & $30(46.9)$ & $51(100)$ & $38(100)$ \\
\hline Well or moderately differentiated & NA & $22(43.1)$ & $28(73.7)$ \\
\hline Poorly differentiated & NA & $25(49.0)$ & $10(26.3)$ \\
\hline Unknown & 0 & $4(7.8)$ & 0 \\
\hline Squamous cell carcinoma & $23(35.9)$ & 0 & 0 \\
\hline Large cell carcinoma & $2(3.1)$ & 0 & 0 \\
\hline Unclassifiable & $9(14.1)$ & 0 & 0 \\
\hline \multicolumn{4}{|l|}{ Measurable target lesions } \\
\hline Lungs & $63(98.4)$ & $3(5.9)$ & $9(23.7)$ \\
\hline Lymph nodes & $33(51.6)$ & $28(54.9)$ & $10(26.3)$ \\
\hline Liver & $9(14.1)$ & $30(58.8)$ & $30(78.9)$ \\
\hline Adrenal glands & $3(4.7)$ & 0 & 0 \\
\hline Bones & $3(4.7)$ & 0 & $1(2.6)$ \\
\hline Pancreas & $1(1.6)$ & $2(3.9)$ & $1(2.6)$ \\
\hline Peritoneum & 0 & $1(2.0)$ & $4(10.5 \%)$ \\
\hline Ovary & 0 & 0 & $1(2.6)$ \\
\hline \multicolumn{4}{|l|}{ First-line chemotherapy } \\
\hline Taxane $+/$ - platinum & $27(42.2)$ & 7 (13.7) & 0 \\
\hline Gemcitabine +/- platinum & $14(21.9)$ & 0 & 0 \\
\hline Irinotecan $+/$ - platinum & $7(10.9)$ & 0 & 0 \\
\hline Pemetrexed $+/$ - platinum & $6(9.4)$ & 0 & 0 \\
\hline Etoposide $+/$ - platinum & $4(6.2)$ & 0 & 0 \\
\hline EGFR-TKI (gefitinib or erlotinib) & $6(9.4)$ & 0 & 0 \\
\hline FOLFOX & 0 & $24(47.1)$ & $33(86.8)$ \\
\hline FOLFIRI & 0 & $2(3.9)$ & $5(13.2)$ \\
\hline TS- 1 or capecitabine $+/$ - cisplatin & 0 & $13(25.5)$ & 0 \\
\hline 5-fluorouracil + cisplatin & 0 & $2(3.9)$ & 0 \\
\hline Docetaxel + oxaliplatin + TS-1 & 0 & $6(11.8)$ & 0 \\
\hline
\end{tabular}

Abbreviations: NSCLC, non-small cell lung cancer; GC, gastric cancer; CRC, colorectal cancer; NA, not available; EGFR-TKI, epidermal growth factor receptor inhibitor; FOLFOX, oxaliplatin plus 5-fluououracil/leucovorin; FOLFIRI, Irinotecan plus 5-fluorouracil/leucovorin.

Among 64 patients with advanced or metastatic NSCLC [13], most patients $(92.2 \%)$ were diagnosed with stage IV NSCLC and only five had an advanced disease (stage IIIB). Thirty patients (46.9\%) had adenocarcinoma and twenty-three (35.9\%) had squamous cell carcinoma. Almost all patients $(98.4 \%)$ had at least one measurable lesion in the lungs. Twenty-three patients $(35.9 \%)$ had two target lesions in the lungs according to RECIST 1.1. Except for the lungs, the most common metastatic site with measurable target lesions was the LNs $(51.6 \%)$, followed by the liver $(14.1 \%)$. Twenty-seven (42.2\%) had target lesions in two organs, most commonly in the lungs and LNs. Seven patients $(10.9 \%)$ revealed target lesions in 3 or more organs. As a first-line chemotherapy, twenty-seven patients $(42.2 \%)$ received taxane with or without platinum, and fourteen $(21.9 \%)$ were treated with gemcitabine with or without platinum. Six patients $(9.4 \%)$ received epidermal growth factor receptor inhibitor (EGFR-TKI) such as erlotinib or gefitinib.
Among 51 patients with metastatic GC [14], twenty-five patients $(49.0 \%)$ had poorly differentiated adenocarcinoma. The most common metastatic site with target lesions was the liver $(58.8 \%)$, followed by the LNs (54.9\%). Thirty-nine patients $(76.5 \%)$ had target lesions only in a single organ: liver in 21 and LNs in 18. Eight patients (15.7\%) had target lesions in two organs, most commonly in the liver and LNs. As a first-line chemotherapy, FOLFOX (5-fluorouracil/ leucovorin plus oxaliplatin) $(47.1 \%)$ were the most common regimen, followed by oral fluorouracil (capecitabine or TS-1) with or without cisplatin (25.5\%).

Among 38 patients with metastatic CRC [15], thirty-two patients $(84.2 \%)$ had colon cancer and the remaining 6 had rectal cancer. Twenty-eight patients $(73.7 \%)$ had well or moderately differentiated adenocarcinoma. The most common metastatic site with measurable target lesions was the liver $(78.9 \%)$, followed by the LN $(26.3 \%)$ and lungs $(23.7 \%)$. Twen- 
ty-five patients $(65.8 \%)$ had target lesions according to the RECIST 1.1 only in a single organ, mostly in the liver. Eleven patients $(28.9 \%)$ had target lesions in two organs, most commonly in the liver and LNs. Three patients had target lesions in 3 or more organs. Most patients $(86.8 \%)$ were treated with FOLFOX regimen as a first-line chemotherapy.

\section{Number of target lesions}

Regardless of the primary sites, the number of target lesions according to the mRECIST 1.1 was significantly lower than that according to the RECIST 1.1 $(\mathrm{P}<0.001)$. The median number of target lesions was 2.5 (range, 2-5) by the RECIST 1.1 and 1.5 (range, 1-4) by the mRECIST 1.1, respectively. There was only one patient who had a metastatic site with a newly defined target lesion by adopting the mRECIST 1.1, instead of the RECIST 1.1. The patient with NSCLC had four target lesions with each one in the lung, LN, liver, and bone according to the mRECIST 1.1, while having a total of five target lesion in the lungs (two), LNs (two), and liver (one) according to the RECIST 1.1.

\section{Tumor responses according the RECIST 1.1 versus MRECIST 1.1}

In 64 patients with NSCLC [13], the differences in the percentage changes of the sum of tumor measurements between the RECIST 1.1 and mRECIST 1.1 were all within $10 \%$. The tumor responses showed a high concordance between the two criteria (Table 2), with a kappa value of 0.899 [95\% confidence interval (CI), 0.788 - 1.0]. Only three patients $(4.7 \%)$ showed disagreement of the tumor responses between the two criteria. One patient with PR by $30.4 \%$ decrease of the sum of tumor measurements according to the RECIST 1.1 was reclassified as SD with $25.4 \%$ decrease according to the mRECIST 1.1. Another patient with SD (27.5\% decrease) according to the RECIST 1.1 was reclassified as PR (32.4\% decrease) according to the mRECIST 1.1. The remaining patient showed PD (20.1\% increase) according to the RECIST 1.1 but was re-categorized as SD (16.1\% increase) according to the mRECIST 1.1. The ORRs of first-line chemotherapy were not different between two criteria $(20.3 \%$ versus $20.3 \%, \mathrm{P}=1.0)$.

Among 51 patients with GC [14], 29 patients (56.9\%) showed a reduction (range, 0.3-12.4\%) in the absolute value of the percentage change of the sum of tumor measurements when adopting the mRECIST 1.1, instead of the RECIST 1.1. The biggest difference observed in the percentage changes between the two criteria was $22.9 \%$ (from $27.9 \%$ increase by the RECIST 1.1 to $50.8 \%$ increase by the mRECIST 1.1). The level of concordance in the tumor response assessment between the RECIST 1.1 and mRECIST 1.1 was excellent
(Table 3), with a kappa value of 0.904 (95\% CI, 0.798 1.00). Three patients $(5.9 \%)$ showed disagreement of the tumor responses between the two criteria. When adopting the mRECIST 1.1 instead of the RECIST 1.1, two patients were re-categorized from SD (decreased by $20 \%$ and $25.5 \%$, respectively) to PR (decreased by $35 \%$ and $42.1 \%$, respectively), and the remaining one with PD (26.1\% increase) was reclassified as SD (16.7\% increase). The ORRs were not significantly different between the two criteria $(45.1 \%$ by the RECIST 1.1 versus $49.0 \%$ by the mRECIST 1.1, $\mathrm{P}=0.692$ ).

Among 38 patients with CRC [15], 18 patients $(47.4 \%)$ showed an increase (range, $0.1-14.7 \%$ ) in the absolute value of the percentage change when adopting the mRECIST 1.1. There also was an excellent agreement between the two criteria in the assessment of tumor response (Table 4), with a kappa value of 0.905 (95\% CI, 0.777-1.0). Only two patients $(5.3 \%)$ showed disagreement of the tumor responses between the two criteria. They showed PR (decreased by $31.9 \%$ and $34.9 \%$, respectively) according to the RECIST 1.1 but were reclassified as SD (decreased by $28.6 \%$ and $24.5 \%$, respectively) according to the mRECIST 1.1. The ORRs of first-line chemotherapy were not significantly different between two criteria (39.5\% according to the RECIST 1.1 versus $34.2 \%$ according to the mRECIST 1.1, $\mathrm{P}=0.226$ ).

Table 5 shows the pooled analysis of the tumor response assessment according to the RECIST 1.1 versus mRECIST 1.1 in all 153 patients. There was a high concordance between the two criteria in the assessment of the tumor response, with a kappa value of 0.908 (95\% CI, 0.846 - 0.970). Taken as a whole, eight patients $(5.2 \%)$ showed discordance in the tumor response assessment between the two criteria. Six patients showed disagreement between PR and SD, and two between SD and PD. The ORRs of first-line chemotherapy, which were calculated regardless of the primary sites and anti-cancer treatment, were not significantly different between the two criteria (33.3\% versus $33.3 \%, \mathrm{P}=1.0)$.

Table 2. Tumor responses by RECIST 1.1 versus mRECIST1.1 in patients with advanced non-small cell lung cancer [13].

\begin{tabular}{lllll}
\hline $\begin{array}{l}\text { Tumor response } \\
\text { by RECIST } 1.1\end{array}$ & \multicolumn{2}{l}{$\begin{array}{l}\text { Tumor response } \\
\text { by mRECIST } 1.1\end{array}$} & \multirow{2}{*}{ Total } \\
\cline { 2 - 4 } & CR + PR & SD & PD & 13 \\
\hline CR + PR & 12 & 1 & 0 & 44 \\
SD & 1 & 43 & 0 & 7 \\
PD & 0 & 1 & 6 & 64 \\
Total & 13 & 45 & 6 & \\
\hline
\end{tabular}

The level of concordance of tumor responses between the two criteria is 0.899 (kappa statistics)

The overall response rates were $20.3 \%$ by the RECIST 1.1 and $20.3 \%$ by the mRECIST 1.1 $(\mathrm{P}=1.0)$ 
Table 3. Tumor responses by RECIST 1.1 versus mRECIST1.1 in patients with advanced gastric cancer [14].

\begin{tabular}{lllll}
\hline $\begin{array}{l}\text { Tumor response } \\
\text { by RECIST } 1.1\end{array}$ & \multicolumn{2}{l}{$\begin{array}{l}\text { Tumor response } \\
\text { by mRECIST } 1.1\end{array}$} & \multirow{2}{*}{ Total } \\
\cline { 2 - 4 } & CR+PR & SD & PD & \\
\hline CR + PR & 23 & 0 & 0 & 23 \\
SD & 2 & 18 & 0 & 20 \\
PD & 0 & 1 & 7 & 8 \\
Total & 25 & 19 & 7 & 51 \\
\hline
\end{tabular}

The level of concordance of tumor responses between the two criteria is 0.904 (kappa statistics).

The overall response rates were $45.1 \%$ by the RECIST 1.1 and $49.0 \%$ by the mRECIST $1.1(\mathrm{P}=0.692)$

Table 4. Tumor responses by RECIST 1.1 versus mRECISTI.1 in patients with metastatic colorectal cancer [15].

\begin{tabular}{lllll}
\hline $\begin{array}{l}\text { Tumor response } \\
\text { by RECIST 1.1 }\end{array}$ & \multicolumn{2}{l}{$\begin{array}{l}\text { Tumor response } \\
\text { by mRECIST } 1.1\end{array}$} & Total \\
\cline { 2 - 4 } & CR + PR & SD & PD & \\
\hline CR + PR & 13 & 2 & 0 & 15 \\
SD & 0 & 20 & 0 & 20 \\
PD & 0 & 0 & 3 & 3 \\
Total & 13 & 22 & 3 & 38 \\
\hline
\end{tabular}

The data presented here were modified from the original table because another three patients were newly included, instead of three showing new lesions at follow-up CT.

The level of concordance of tumor responses between the two criteria is 0.905 (kappa statistics)

The overall response rates were $39.5 \%$ by the RECIST 1.1 and $34.2 \%$ by the mRECIST $1.1(\mathrm{P}=0.226)$

Table 5. The pooled analysis of tumor responses by RECIST 1.1 versus $\mathrm{mRECIST} 1.1$ in all patients

\begin{tabular}{lllll}
\hline $\begin{array}{l}\text { Tumor response } \\
\text { by RECIST } 1.1\end{array}$ & \multicolumn{2}{l}{$\begin{array}{l}\text { Tumor response } \\
\text { by mRECIST } 1.1\end{array}$} & \multirow{2}{*}{ Total } \\
\cline { 2 - 4 } & CR + PR & SD & PD & 51 \\
\hline CR + PR & 48 & 3 & 0 & 84 \\
SD & 3 & 81 & 0 & 18 \\
PD & 0 & 2 & 16 & 153 \\
Total & 51 & 86 & 16 & 0.908
\end{tabular}

The level of concordance of tumor responses between the two criteria is 0.908 (kappa statistics).

The overall response rates were $33.3 \%$ by the RECIST 1.1 and $33.3 \%$ by the mRECIST $1.1(\mathrm{P}=1.0)$

\section{Discussion}

We investigated the impact of measuring the single largest lesion in each organ (mRECIST 1.1), instead of two target lesions per organ (RECIST 1.1), on the tumor responses in patients with advanced or metastatic NSCLC, GC, and CRC. As expected, the mRECIST 1.1 significantly reduced the number of target lesions to be assessed for evaluating tumor response after chemotherapy. However, the mRECIST 1.1 has shown a high concordance with the RECIST 1.1 in the assessment of tumor response.

An accurate assessment of objective therapeutic response is essential for routine anti-cancer treatment as well as clinical trials using new drugs. Both WHO and RECIST guidelines depend mainly on the radio- logic changes of tumor size in the assessment of tumor response. It would be ideal if all tumors could be measured, but this is always not possible in clinical practice. Therefore, it is important to choose the most appropriate target lesions that can be accurately measured and followed, reflecting the changes of the overall tumor burden. The RECIST 1.0 recommended a total of ten target lesions with a maximum of five per organ to be measured. Although these target lesions were assumed to be representative of the entire tumor burden in each patient, however, the number of target lesions to be measured was arbitrarily selected, without certain objective evidence. Thereafter the RECIST Working Group retrospectively analyzed the effect of assessing one, two, three or five target lesions, instead of ten, on the tumor response outcome using their patient database [7]. The results indicated that, when compared with assessing ten lesions, assessing three or five target lesions did not changed the ORR and only minimally affected progression-free survival. A statistical simulation model for evaluating the impact of the number of target lesions also revealed no difference between five and ten target lesions in the assessment of tumor response [10]. Based on these results, the RECIST 1.1 adopted a total of five target lesions to be measured, with a maximum of two lesions per organ.

The RECIST 1.1 have shown almost perfect concordance with the RECIST 1.0 in the assessment of tumor response in patients with NSCLC $[16,17]$, advanced GC [18,19], and metastatic CRC [20]. With an expectation of improving feasibility via more convenient assessment of tumor response, the RECIST 1.1 has rapidly adopted in clinical practice as well as clinical trials. To the best of our knowledge, however, the criterion of two target lesions per organ in the RECIST 1.1 was still an arbitrary value and has been supported by no objective evidence. Furthermore, in patients with more than three metastatic sites, the RECIST 1.1 may not be representative of all involved organs because of the limited total number of target lesions assessed. Herein we assumed that measuring the single largest lesion in each organ could represent more metastatic sites. Contrary to our expectation, however, only one patient was identified to have an additional metastatic site with a newly emerging target lesion by adopting the mRECIST 1.1.

There have been only a few studies in the literature investigating the optimal number of target lesions to be measured in order to evaluate tumor response. Schwartz et al. [11] simulated more than 1.8 million possible combinations of target lesions using a complex computerized model. The results indicated that the average variance in the assessment of tumor response was decreased by $90 \%$ if four lesions were 
uni-dimensionally measured instead of only one. Darkeh et al. [21] investigated the minimum number of target lesions needed to be representative of the total number of target lesions according to the RECIST 1.0. In 53 patients with five or more target lesions, measuring four to seven lesions (whenever applicable) led to no discrepancy. However, the percentage of discordant cases increased gradually from measuring three lesions $(5.5 \%)$ to measuring one target lesion (15.1\%). Based on these results, they recommended measuring at least four lesions if patients had more than four target lesions. However, these studies only investigated the minimum number of target lesions to be measured, without considering the optimal number of target lesions per organ.

There is a single study comparing the value of measuring a solitary well-defined lesion with multiple-lesion measurements in the literature. Prior to the publication of the RECIST 1.1, Zacharia et al. compared the tumor responses between the single-lesion measurement and multiple-lesion measurement in patients undergoing chemotherapy for colon cancer metastases to the liver [12]. Measuring two or more target lesions up to five showed a perfect concordance in the assessment of the tumor response. However, the single-lesion measurements also yielded the same response classification in $93.3 \%$ of patients $(28 / 30)$, with a kappa value of 0.88 when compared with the multiple-lesion measurements. This finding suggested that it might be possible to measure the single largest lesion per organ for assessing tumor response.

Recently, we compared the tumor responses by CT between the RECIST 1.1 and mRECIST 1.1 in patients with advanced or metastatic NSCLC [13], GC [14], and CRC [15]. The mRECIST 1.1, with a decreased total number of target lesions to be measured, was comparable to the original RECIST 1.1 in the assessment of tumor response, with a kappa value of $0.899,0.904$, and 0.905 respectively. In the current study, we conducted a pooled analysis using the data from the three studies to improve statistical power. When comparing tumor response in a total of 153 patients who had at least two target lesions in any organ based on the RECIST 1.1, the level of concordance in the tumor responses between the RECIST 1.1 and mRECIST 1.1 was very high, with a kappa value of 0.908 . This kappa value is slightly higher than the individual value in each type of tumor. Eight patients $(5.2 \%)$ showed discordance in the tumor response assessment between the two criteria: six between PR and SD; two between SD and PD. By adopting the mRECIST 1.1 instead of the RECIST 1.1, five patients were upgraded (three from SD to PR, and two from $\mathrm{PD}$ to SD) and three were downgraded from PR to SD in the assessment of the tumor response. Traditional- ly, patients who achieve PR or SD remain on the same treatment regimen in clinical practice. In the current study with only two patients showing discordance between PD and SD, therefore, the clinical impact of the mRECIST 1.1 on altering therapeutic plan appeared to be minimal.

The statistical simulation study for evaluating the impact of the number of lesions reported that a smaller number of target lesions tended to overestimate the response rate [10]. In this pooled analysis, however, the ORRs estimated regardless of the primary site and anti-cancer treatment were not significantly different between the two criteria $(33.3 \%$ by the RECIST 1.1 versus $33.3 \%$ by the RECIST $1.0, P=1.0$ ). In clinical practice, although it is very rare, patients can exhibit mixed tumor responses in which some tumors shrink whereas others grow [22-24]. This phenomenon may be explained by inter-tumor heterogeneity in the mutational status [24]. Theoretically, mixed responses may lead to discordance between the two response criteria. Among eight patients who revealed discordance between the RECIST 1.1 and mRECIST 1.1, however, none exhibited mixed tumor response. Therefore selecting a single target lesion did not appear to grossly overestimate or underestimate tumor response in the current study.

There is a concern that the largest lesion may not always be the best target lesion. Large lesions may be partially necrotic or contain cavitations may not shrink to the same extent as smaller lesions that respond to treatment [25]. In those cases, however, the RECIST 1.1 which measure two largest lesions per organ also has a potential risk that do not exactly reflect the changes of tumor burden. The functional image methods such as diffusion-weighted magnetic resonance (MR) imaging or dynamic contrast-enhanced imaging with CT or MR may need to be incorporated for target lesions with necrosis or cavities [26].

On the assumption that the mRECIST 1.1 is comparable to the RECIST 1.1 in the assessment of tumor response, the single-lesion measurement per organ has a couple of advantages over the two-lesion measurements. In this study, the number of target lesions according to the mRECIST 1.1 was significantly lower than that according to the RECIST 1.1 ( $P$ $<0.001$ ). Therefore, the mRECIST 1.1, with a decreased total number of target lesions, is expected to not only increase convenience but also decrease intraand inter-observer variability in the measurement of target lesions. In addition, because the highest number of target lesions according to the mRECIST 1.1 was 4 , the mRECIST 1.1 may not need to limit the maximum number of target lesions.

This pooled analysis has several limitations 
needed to be described. First, this study was conducted only in patients with three types of malignancies. It means that our results are insufficient to be generalized for patients with primary cancer in other sites. Especially, patients with metastatic breast cancer or prostatic cancer develop frequently bone metastases during their disease course. As mentioned earlier, lytic or mixed lytic-blastic bone lesions with an identifiable soft tissue component can be regarded as a target lesion according to the RECIST 1.1. Therefore, the mRECIST 1.1 need to be investigated in patients with breast cancer or prostatic cancer. Second, most patients in this study received a first-line treatment with cytotoxic agents. A number of recently developed target agents induce necrosis and cystic change in solid tumors without necessarily producing tumor shrinkage. The single-lesion measurement in each organ needs to be verified in patients receiving target agents. Third, although the mRECIST 1.1 is comparable to the RECIST 1.1 in the assessment of tumor response, it may differently affect time to progression, which was not evaluated in this study.

In conclusion, the modified RECIST 1.1, despite the significantly decreased total number of target lesions to be measured, was comparable to the original RECIST 1.1 in the assessment of tumor response. Our result suggests that it might be possible to measure the single largest target lesion per organ for assessing tumor response. However, further confirmatory studies with larger series of patients are warranted to validate our finding.

\section{Competing Interests}

The authors have declared that no competing interest exists.

\section{References}

1. Miller AB, Hoogstraten B, Staquet M, Winkler A. Reporting results of cancer treatment. Cancer. 1981; 47: 207-14.

2. Thiesse P, Ollivier L, Di Stefano-Louineau D, Négrier S, Savary J, Pignard K, et al. Response rate accuracy in oncology trials: reasons for interobserver variability. Groupe Français d'Immunothérapie of the Fédération Nationale des Centres de Lutte Contre le Cancer. J Clin Oncol. 1997;15:3507-14.

3. Choi JH, Ahn MJ, Rhim HC, Kim JW, Lee GH, Lee YY, et al. Comparison of WHO and RECIST criteria for response in metastatic colorectal cancer. Cancer Res Treat. 2005;37:290-3.

4. Therasse P, Arbuck SG, Eisenhauer EA, Wanders J, Kaplan RS, Rubinstein L, et al. New guidelines to evaluate the response to treatment in solid tumors. European Organization for Research and Treatment of Cancer, National Cancer Institute of the United States, National Cancer Institute of Canada. J Natl Cancer Inst. 2000;92:205-16.

5. Sargent DJ, Rubinstein L, Schwartz L, Dancey JE, Gatsonis C, Dodd LE, et al. Validation of novel imaging methodologies for use as cancer clinical trial endpoints. Eur J Cancer. 2009;45: 290-9.

6. Eisenhauer EA, Therasse P, Bogaerts J, Schwartz LH, Sargent D, Ford R, et al. New response evaluation criteria in solid tumours: revised RECIST guideline (version1.1). Eur J Cancer. 2009;45:228-47.

7. Bogaerts J, Ford R, Sargent D, Schwartz LH, Rubinstein L, Lacombe D, et al. Individual patient data analysis to assess modifications to the RECIST criteria. Eur J Cancer. 2009;45:248-60.

8. Schwartz LH, Bogaerts J, Ford R, Shankar L, Therasse P, Gwyther S, et al. Evaluation of lymph nodes with RECIST 1.1. Eur J Cancer. 2009;45:261-7.

9. Dancey JE, Dodd LE, Ford R, Kaplan R, Mooney M, Rubinstein L, et al. Recommendations for the assessment of progression in randomized cancer treatment trials. Eur J Cancer. 2009;45:281-9.
10. Moskowitz CS, Jia X, Schwartz LH, Gonen M. A simulation study to evaluate the impact of the number of lesions measured on response assessment. Eur J Cancer. 2009;45:300-10.

11. Schwartz LH, Mazumdar M, Brown W, Smith A, Panicek DM. Variability in response assessment in solid tumors: Effect of number of lesions chosen for measurement. Clin Cancer Res. 2003;9:4318-23.

12. Zacharia TT, Saini S, Halpern EF, Sumner JE. CT of colon cancer metastases to the liver using modified RECIST criteria: determining the ideal number of target lesions to measure. AJR Am J Roentgenol. 2006;186:1067-70.

13. Kim HS, Kim JH, Yang IK. Tumor response assessment by measuring the single largest lesion per organ in patients with advanced non-small cell lung cancer. Lung Cancer. 2014;85:385-9.

14. Kim HS, Kim JW, Kim JH, Choi DR, Kim M-J, Han AR, et al. The single-lesion measurement for assessing tumor response in patients advanced gastric cancer. Oncology. 2015;88:69-75.

15. Kim HS, Kim JH. Ideal number of target lesions per organ to measure in metastatic colorectal cancer. Oncol Lett. 2014;8:1896-900.

16. Sun JM, Ahn MJ, Park MJ, Yi JH, Kim TS, Chung MJ, at al. Accuracy of RECIST 1.1 for non-small cell lung cancer treated with EGFR tyrosine kinase inhibitors. Lung Cancer. 2010;69:105-9.

17. Nishino M, Jackman DM, Hatabu H, Yeap BY, Cioffredi LA, Yap JT, et al. New Response Evaluation Criteria in Solid Tumors (RECIST) guidelines for advanced non-small cell lung cancer: comparison with original RECIST and impact on assessment of tumor response to target therapy. AJR Am J Roentgenol. 2010;195:221-8.

18. Fuse N, Nagahisa-Oku E, Doi T, Sasaki T, Nomura S, Kojima T. Effect of RECIST revision on classification of target lesions and overall response in advanced gastric cancer patients. Gastric Cancer. 2013;16:324-8.

19. Jang GS, Kim MJ, Ha HI, Kim JH, Kim HS, Ju SB, et al. Comparison of RECIST version 1.0 and 1.1 in assessment of tumor response by computed tomography in advanced gastric cancer. Chin J Cancer Res. 2013;25:689-94.

20. Jang HJ, Kim BC, Kim HS, Kim JH, Song HH, Kim JB, et al. Comparison of RECIST 1.0 and RECIST 1.1 on computed tomography in patients with metastatic colorectal cancer. Oncology. 2014;86:117-21.

21. Darkeh MH, Suzuki C, Torkzad MR. The minimum number of target lesions that need to be measured to be representative of the total number of target lesions (according to RECIST). Br J Radiol. 2009;82:681-6.

22. Ryoo BY, Na II, Yang SH, Koh JS, Kim CH, Lee JC. Synchronous multiple primary lung cancers with different response to gefitinib. Lung Cancer. 2006;53:245-58.

23. Park KY, Jung JW, Nam SB, Lee HJ, Kim HS, Choi YH, Lee JC. Two lung masses with different responses to pemetrexed. Korean J Intern Med. 2010;25:213-6.

24. Chen ZY, Zhong WZ, Zhang XC, Su J, Yang XN, Chen ZH, et al. EGFR mutation heterogeneity and the mixed response to EGFR tyrosine kinase inhibitors of lung adenocarcinomas. Oncologist. 2012;17:978-85.

25. Nishino M, Jagannathan JP, Ramaiya NH, Van den Abbeele Ad. Revised RECIST guideline version 1.1: what oncologists want to know and what radilologist need to know. AJR Am J Roentgenol. 2010;195:281-9.

26. Desar IM, van Herpen CM, van Laarhoven HW, Barentsz JO, Oyen WJ, van der Graaf WT. Beyond RECIST: molecular and functional imaging techniques for evaluation of response to targeted therapy. Cancer Treat Rev. 2009;35:309-21. 